\title{
Foxc2 and BMP2 Induce Osteogenic/Odontogenic Differentiation and Mineralization of Human Stem Cells from Apical Papilla
}

\author{
Wen Zhang $\mathbb{D}^{1},{ }^{1}$ Xiaolei Zhang, ${ }^{1}$ Junyuan $\mathrm{Li}^{2}{ }^{2}$ Jianmao Zheng, ${ }^{1}$ Xiaoli Hu, ${ }^{1}$ Meng Xu, \\ Xueli Mao, ${ }^{1}$ and Junqi Ling $\mathbb{1}^{1}$ \\ ${ }^{1}$ Guangdong Province Key Laboratory of Stomatology, Guanghua School and Hospital of Stomatology, Sun Yat-sen University, \\ Guangzhou, Guangdong 510055, China \\ ${ }^{2}$ The Medical Centre of Stomatology, The 1st Affiliated Hospital of Jinan University, Guangzhou 510630, China
}

Correspondence should be addressed to Junqi Ling; lingjq@mail.sysu.edu.cn

Received 17 March 2018; Accepted 24 June 2018; Published 25 July 2018

Academic Editor: Daniel Bouvard

Copyright ( 2018 Wen Zhang et al. This is an open access article distributed under the Creative Commons Attribution License, which permits unrestricted use, distribution, and reproduction in any medium, provided the original work is properly cited.

\begin{abstract}
As a transcription factor regulated by bone morphogenetic protein 2 (BMP2), Forkhead c2 (Foxc2) plays a pivot role in osteogenesis/odontogenesis. However, the role of Foxc2 and BMP2 in regulating osteo-/odontogenic differentiation and mineralization of stem cells from apical papilla (SCAP) is still uncertain. In this research, overexpression of Foxc2 gene significantly improved the proliferation of SCAP four days and eight days after transfection, but overexpression of both Foxc2 and BMP2 genes significantly inhibited the proliferation of SCAP eight days after transfection. RT-qPCR and western blot results indicated that SCAP-Foxc2-BMP2 significantly upregulated osteo-/odontogenic genes and proteins at most of the time points in SCAP after transfection. Moreover, SCAP-Foxc2-BMP2 formed notably more alkaline phosphatase-positive and alizarin red-positive mineralized nodules than other three group cells sixteen days after transfection. In conclusion, our findings revealed that Foxc2 and BMP2 synergistically promoted osteo-/odontogenic differentiation and mineralization of SCAP in vitro.
\end{abstract}

\section{Introduction}

How to effectively regenerate dentin and repair tooth defects is the key problem in dental regenerative medicine [1]. Tissue engineering dentin is the use of a combination of cells, engineering and material methods, and suitable biochemical and physicochemical factors to regenerate biological dentin. Human stem cells from apical papilla (SCAP) are considered to be suitable seed cells for dentin regeneration, because they have osteo-/odontogenic differentiation potential, good individual specificity, and high proliferative activity [2]. Moreover, they can regularly be obtained from extracted immature third molars or orthodontic teeth in clinical practice [3]. However, without induction, the capability of forming mineralized structure/ tissue by SCAP is insufficient [4-6]. Thus, it is necessary to explore the key genes in regulating osteo-/odontogenic differentiation of SCAP and on this basis enhance their expression in order to enable reparative dentin formation.
Dentin development is the result of interaction between dental epithelium and mesenchyme, and multiple molecular pathways are involved in regulating this process [7]. Bone morphogenetic protein (BMP) family, a part of the transforming growth factor beta (TGF- $\beta$ ) superfamily, is crucial in dental papilla growth and development $[7,8]$. BMP2 is one of the strongest osteo-/odontogenic induction members in the BMP family, supporting osteo-/odontogenic differentiation of dental pulp stem cells $[4,6,9]$. Wu et al. reported that BMP2-containing medium could promote SCAP to form dentin-like tissue [10]. In our previous studies [4, 6], BMP2 gene-modified SCAP (SCAP-BMP2) presented significantly upregulated osteo-/odontogenic differentiation genes/proteins. Thus, for osteogenic or odontogenic differentiation of SCAP, BMP2 is an essential target gene.

Forkhead c2 (Foxc2) is a member of the winged spiral transcription factor family. Foxc2 participates in promoting cell proliferation [11, 12], osteogenesis [11, 13-15], and angiogenesis [16]. Moreover, Foxc2 gene-modified 
mesenchymal stem cells expressed osteo-/odontogenic differentiation-related genes, such as alkaline phosphatase (ALP), osteocalcin (OCN), dentin sialophosphoprotein (DSPP), and dentin matrix protein 1 (DMP1) [11, 13-18]. Latest studies pointed out that Foxc2 was highly expressed in developing dental tissue, including dental papilla [19-21], so it is reasonable to speculate that Foxc2 may participate in the differentiation of SCAP.

In the current research, we constructed Foxc2 and BMP2 gene-modified SCAP by lentivirus transfection technique and then investigated the synergistic effects of Foxc2 and BMP2 in inducing osteogenic and odontogenic differentiation of human SCAP.

\section{Methods}

2.1. SCAP Isolation and Identification. A few sound mandibular 3rd molars $(n=12)$ were collected from young patients (18 to 20 years old) in the Oral and Maxillofacial Surgery Department, Sun Yat-sen University. The experiment protocol of the current research was proven by the Ethical Review Committee of Sun Yat-sen University. Freshly extracted third molars were rinsed by PBS, and the apical papillae were isolated by surgical instruments. Sequentially, SCAP were minced and treated with enzymatic digestion according to our previous reports $[4,6]$. Osteogenic and adipogenic differentiation of SCAP was analyzed by alizarin red staining and Oil Red staining, respectively. The SCAP phenotypic markers, such as STRO-1 (Santa Cruz, Delaware, CA), CD146 (BD, Pharmingen, USA), CD24 (BD, Pharmingen, USA), and CD45 (BD, Pharmingen, USA), were examined by flow cytometry.

2.2. Lentiviral Plasmid Transfection. Human Foxc2 and BMP2 gene primers, as showed in Table 1, were amplified. Then, the amplified oligonucleotides were merged into the blank vector pCDH-CMV-MCS-EF1-copGFP (System Biosciences, USA) to construct lentiviral recombinant plasmids pCDH-Foxc2 and pCDH-BMP2 according to our previous description $[4,6,22]$. Subsequently, the recombinant lentiviral plasmids, envelop plasmid, and packaging plasmid were transduced into 293FT cells. Forty-eight hours after cell culture, the 293FT cell supernatant was collected. The supernatant was used to infect the 3rd passage SCAP to obtain gene-modified cells-that is, SCAP-Foxc2 and SCAP-BMP2. Similarly, SCAP-Foxc2-BMP2 cells were constructed by infecting SCAP with the Foxc2 lentiviral supernatant and BMP2 lentiviral supernatant. SCAP labelled with GFP (SCAP-GFP) was used as the control. Four days after transfection, the expressions of Foxc2 and BMP2 in the four groups, that is, SCAP-GFP, SCAP-Foxc2, SCAP-BMP2, and SCAP-Foxc2-BMP2, were evaluated by RT-qPCR and western blot analysis.

2.3. Cell Proliferation. Four group cells were seeded on 96well plates with initial density of $2 \times 10^{3}$ cells/well and then cultured with $\alpha$-MEM medium supplied with $10 \%$ FBS. Cell Counting Kit 8 (CCK8, Dojindo, Tokyo, Japan) was applied
TABLE 1: Real-time PCR primers.

\begin{tabular}{|c|c|c|}
\hline Genes & Sequence & Size (bp) \\
\hline BMP2 & $\begin{array}{c}\text { Forward: } 5^{\prime} \text {-ACCCGCTGTCTTCTAG } \\
\text { CGT-3 }{ }^{\prime} \\
\text { Reverse: } 5^{\prime} \text {-TTTCAGGCCGAACATG } \\
\text { CTGAG-3' }\end{array}$ & 180 \\
\hline Foxc2 & $\begin{array}{c}\text { Forward: } 5^{\prime} \text {-CCTCCTGGTATCTCAA } \\
\text { CCACA-3' } \\
\text { Reverse: } 5^{\prime} \text {-GAGGGTCGAGTTCTCA } \\
\text { ATCCC- } 3^{\prime}\end{array}$ & 134 \\
\hline ALP & $\begin{array}{c}\text { Forward: } 5^{\prime} \text {-CTATCCTGGCTCCGTG } \\
\text { CTC- } 3^{\prime} \\
\text { Reverse: } 5^{\prime} \text {-GCTGGCAGTGGTCAGA } \\
\text { TGTT-3' }\end{array}$ & 100 \\
\hline OCN & $\begin{array}{c}\text { Forward: } 5^{\prime} \text {-CTCACACTCCTCGCCC } \\
\text { TATT- } 3^{\prime} \\
\text { Reverse: } 5^{\prime} \text {-TTGGACACAAAGGCTG } \\
\text { CAC- } 3^{\prime}\end{array}$ & 107 \\
\hline DSPP & $\begin{array}{c}\text { Forward: } 5^{\prime} \text {-GCCACTTTCAGTCTTC } \\
\text { AAAGAGA-3' } \\
\text { Reverse: } 5^{\prime} \text {-GCCCAAATGCAAAAAT } \\
\text { ATGTAA- } 3^{\prime}\end{array}$ & 130 \\
\hline DMP1 & $\begin{array}{c}\text { Forward: } 5^{\prime} \text {-AAAATTCTTTGTGAAC } \\
\text { TACGGAGG- } 3^{\prime} \\
\text { Reverse: } 5^{\prime} \text {-GAGCACAGGATAATCC } \\
\text { CCAA- } 3^{\prime}\end{array}$ & 94 \\
\hline GAPDH & $\begin{array}{c}\text { Forward: } 5^{\prime} \text {-AAGGTGAAGGTCGGAG } \\
\text { TCAA-3 }{ }^{\prime} \\
\text { Reverse: } 5^{\prime} \text {-AATGAAGGGGTCATTG } \\
\text { ATGG-3 }{ }^{\prime}\end{array}$ & 108 \\
\hline
\end{tabular}

to analyze the proliferation of those cells on the 1 st, 2 nd, 4 th, and 8th days after transfection.

2.4. RT-qPCR Analysis. Total RNA was extracted by TRIzol (Invitrogen, USA) from the four group cells on the 1st, 4th, 8th, and 16th days after lentiviral transfection. The cDNA synthesis was performed by Revert Aid first strand cDNA synthesis kit (Invitrogen, USA). RT-qPCR reactions were conducted by iQ SYBR Green Supermix (BioRad Laboratories, USA) and regulated by spectrofluorimetric iQ5 thermal iCycler (BioRad Laboratories, USA). The PCR primer sequences were presented in Table 1 . The mRNA expressions of Foxc2, BMP2, ALP, OCN, DSPP, and DMP1 in the four group cells were tested by RT-qPCR at different time points. GAPDH was set as the control.

2.5. Western Blot. First, proteins were extracted from the four group cells, and then proteins were separated by $12 \%$ sodium dodecyl sulfate polyacrylamide gels (Beyotime Institute of Biotechnology, China). The separated proteins 


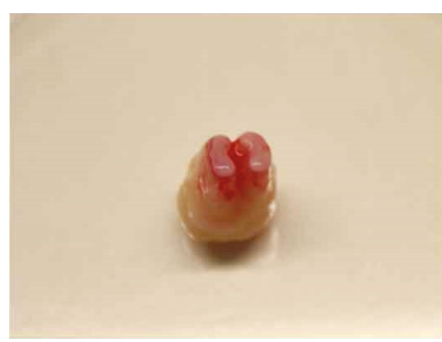

(a)

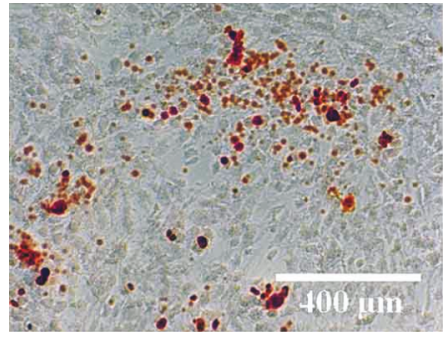

(c)
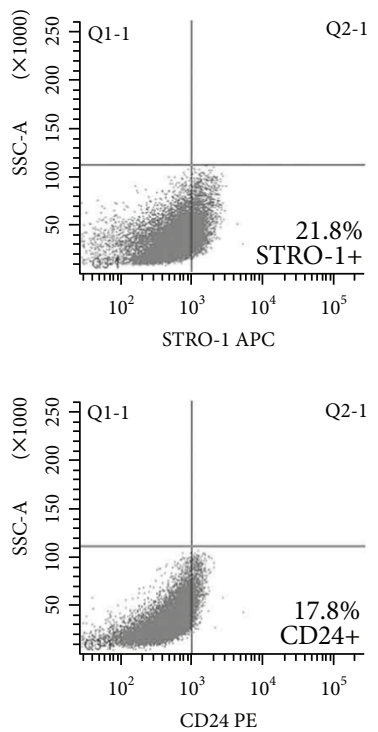

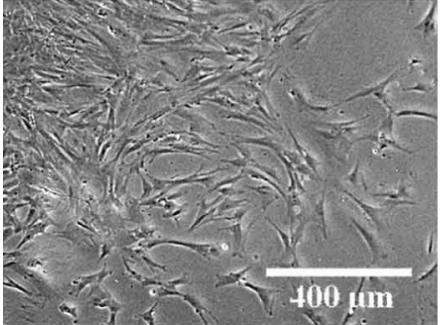

(b)

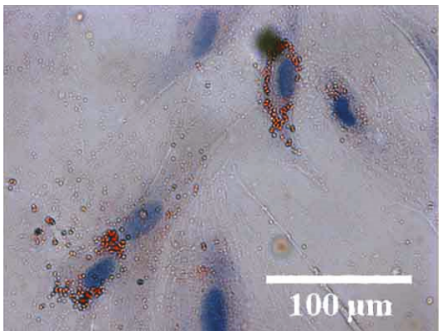

(d)
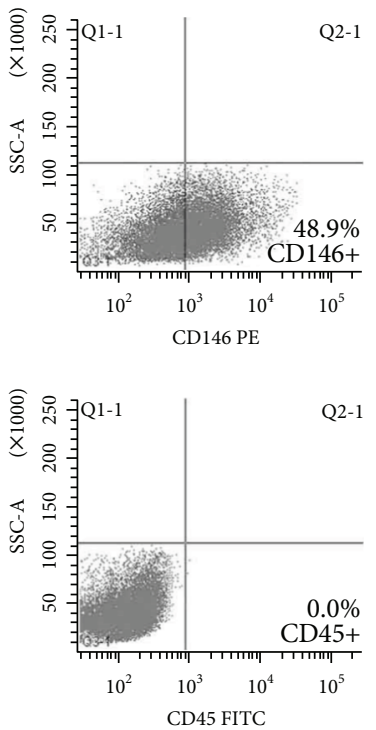

(e)

FIGURE 1: SCAP isolation, culture, and identification. (a) The dental papillae were located at the apical part of the molars and showed pink in color. (b) The primary SCAP were isolated from the dental papilla tissues and displayed spindle-like morphological characters. (c) The 3rd passage SCAP were induced with osteogenic induction medium for 32 days and showed alizarin red-positive mineralized nodules. (d) The 3rd passage SCAP were induced with adipogenic induction medium for 16 days and showed oil red-positive lipid droplets. (e) The 3rd passage SCAP were analyzed by flow cytometry. Those SCAP presented positive expression of STRO-1, CD146, and CD24, but the expression of CD45 was negative.

were then transferred onto PVDF membranes (Thermo Fisher Scientific, USA) at $200 \mathrm{~mA}$ for 2 hours. The $5 \%$ nonfat milk was used to block the PVDF membranes for 2 hours. Subsequently, the PVDF membranes were incubated with the primary antibodies overnight at $4^{\circ} \mathrm{C}$. The primary antibodies include rabbit polyclonal anti-foxc2 (1:500; cat. number ab24340; Abcam, USA), rabbit polyclonal anti-BMP2 (1:500; cat. number ab14933; Abcam), and rabbit polyclonal anti-dentin sialoprotein (DSP; 1:500; cat. number sc-33586; Santa Cruz Biotechnology, USA). After that, the PVDF membranes were incubated with a horseradish peroxidase-conjugated goat anti-rabbit antibody (1:20,000; cat. number ab97051; Abcam) at $37^{\circ} \mathrm{C}$ for 2 hours. The rabbit polyclonal anti-GAPDH $(1: 2500$; cat. number ab9485; Abcam) was chosen as the control. The chemiluminescence western blotting detection system (EMD Millipore) was used to visualize the resultant bands.

2.6. ALP and Alizarin Red Staining. The four group cells were seeded on 6-well plates with the density of $5 \times 10^{4}$ cells/well. ALP kit (Jiancheng Biotech, Nanjing, China) and alizarin 


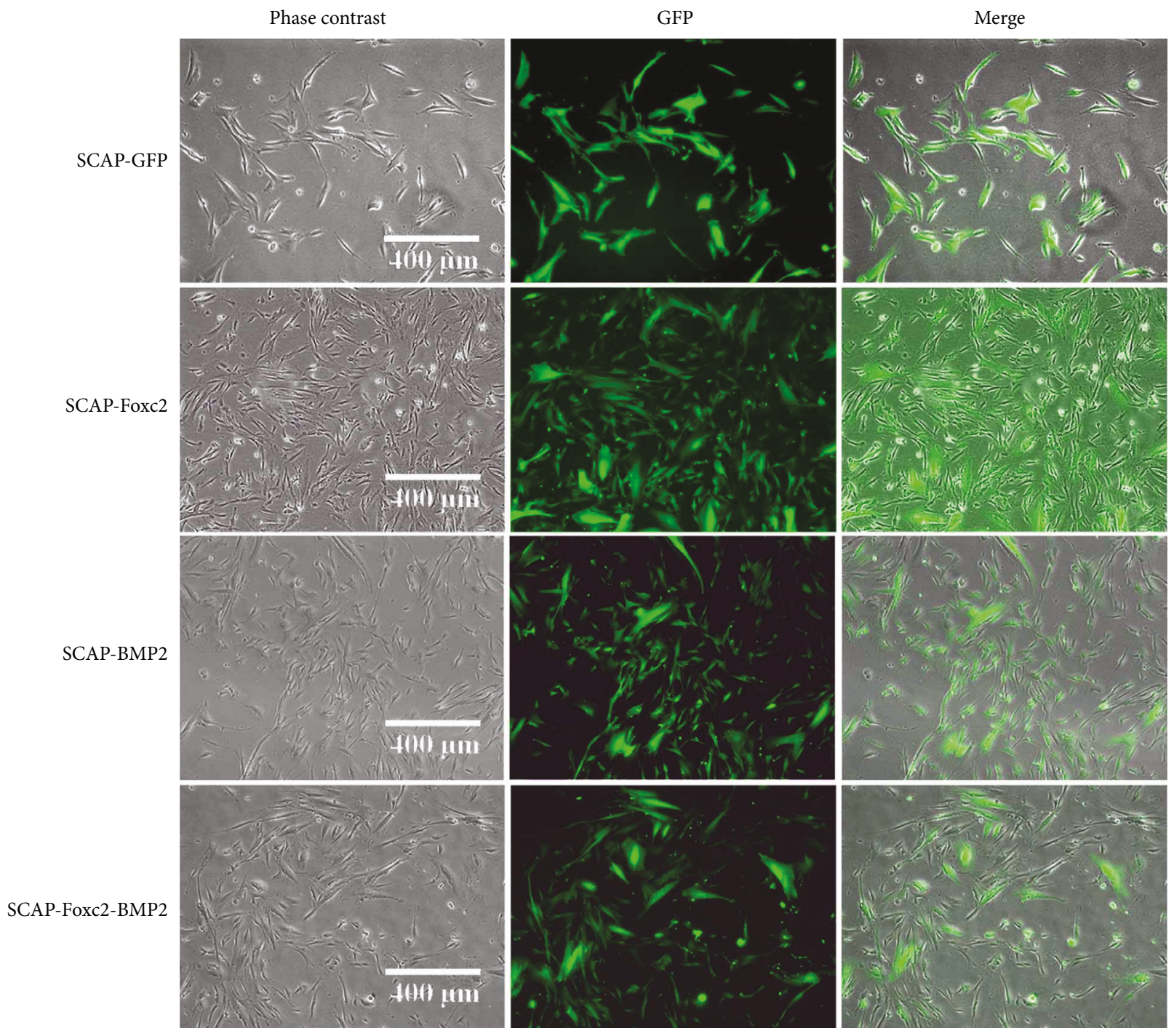

(a)

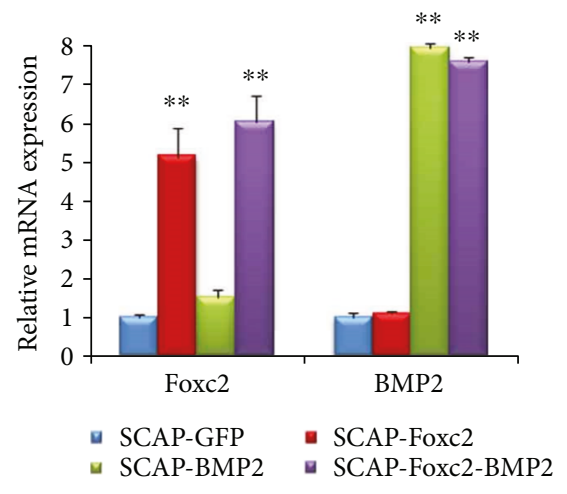

(b)

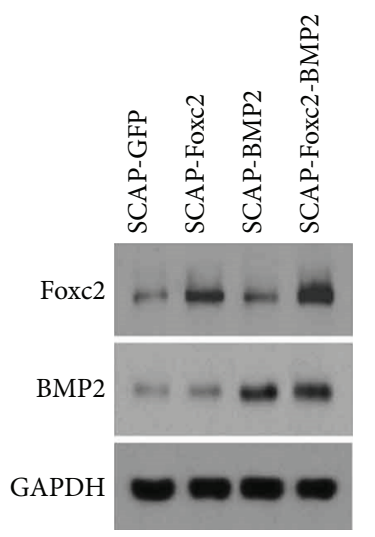

(c)

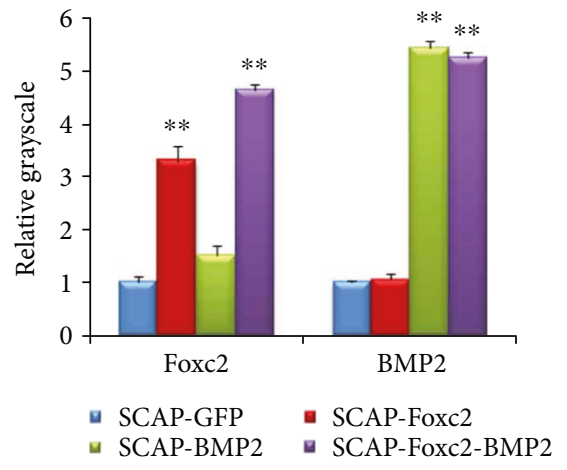

(d)

Figure 2: Lentiviral transfection efficiency. (a) Forty-eight hours after lentiviral transfection, all four group cells presented more than $90 \%$ GFP expression percentage, which means the lentivirus plasmid-mediated gene transfection efficiencies of the four groups cells are high. (b) Four days after transfection, the Foxc2 expression in the mRNA level was significantly upregulated $(P<0.01)$ in SCAP-Foxc2 and SCAP-Foxc2-BMP2. Similarly, the BMP2 expression in the mRNA level was significantly upregulated $(P<0.01)$ in SCAP-BMP2 and SCAP-Foxc2-BMP2. (c, d) Four days after transfection, western blot results further confirmed that the Foxc2 expression in the peptide level was correspondingly increased $(P<0.01)$ in SCAP-Foxc2 and SCAP-Foxc2-BMP2. The BMP2 expression in the peptide level was also significantly $(P<0.01)$ increased in SCAP-BMP2 and SCAP-Foxc2-BMP2. ${ }^{* *} P<0.01$. 


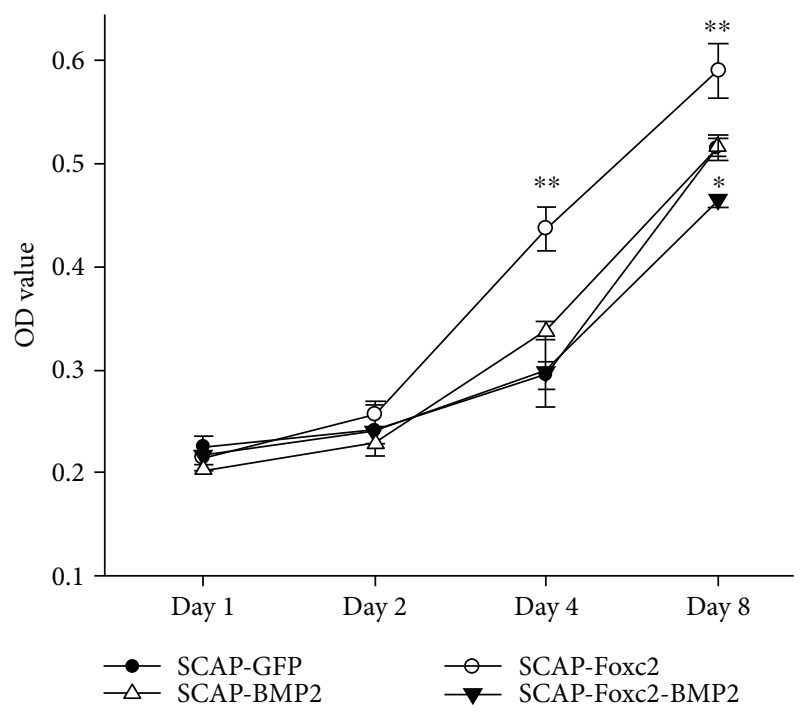

Figure 3: Proliferation status of four group cells. SCAP-Foxc2 demonstrated a significantly higher $(P<0.01)$ proliferation status than the other three group cells on day 4 and day 8 , while the proliferation status of SCAP-Foxc2-BMP2 was significantly weaker $(P<0.05)$ than that of the other three group cells on day 8. ${ }^{*} P<0.05,{ }^{* *} P<0.01$.

red kit (Jiancheng Biotech, Nanjing, China) were applied to the cells to visualize ALP expression and mineralized granules on the day 16.

2.7. Statistical Analysis. One-way ANOVA test was performed to analyze the differences of the four group cells by SPSS 20.0. Statistical significance was set as $P$ value $<0.05$.

\section{Results}

3.1. Characterization of SCAP. The papillae, as showed in Figure 1(a), were pink and kidney-shaped. Those primary SCAP isolated from the apical papillae presented classic cell colonies eight days after culture, and most of the SCAP were spindle-like in shape (Figure 1(b)). SCAP formed many alizarin red-positive mineralized nodules after thirty-two days of osteogenic induction (Figure 1(c)) and formed a few oil red O-positive lipid droplets sixteen days after adipogenic induction (Figure 1(d)). The 3rd passage SCAP displayed positive phenotypic markers, such as STRO-1, CD146, and CD24, while CD45 is negative (Figure 1(e)).

3.2. Expression of Foxc2 and BMP2 in the Gene-Transfected $S C A P$. The cells in the four groups showed very high GFP expression percentage four days after transfection (Figure 2(a)). The relative mRNA expression of Foxc2 in SCAP-GFP, SCAP-Foxc2, SCAP-BMP2, and SCAP-Foxc2$\mathrm{BMP} 2$ was $1.00 \pm 0.05,5.14 \pm 0.72,1.52 \pm 0.18$, and $6.13 \pm 0.69$, respectively, while the relative mRNA expression of BMP2 in the four group cells was $1.00 \pm 0.13,1.11 \pm 0.05$, $7.94 \pm 0.12$, and $7.60 \pm 0.10$, respectively (Figure $2(\mathrm{~b})$ ). The relative Foxc2 peptide expression in the four group cells was $1.00 \pm 0.11,3.29 \pm 0.27,1.52 \pm 0.16$, and $4.63 \pm 0.10$, respectively. The relative BMP2 peptide expressions in the four group cells were $1.00 \pm 0.05,1.07 \pm 0.11,5.42 \pm 0.15$, and $5.23 \pm 0.10$, respectively (Figures $2(\mathrm{c}$ ) and 2(d)). Interestingly, current results presented that BMP2 gene transfection slightly enhanced the mRNA and peptide expression of Foxc2, but the enhancement was not significant $(P>0.05)$.

3.3. Foxc2 and BMP2 Regulate the Proliferation of SCAP. As showed in Figure 3, there were no significant differences of the four group cells on the 1st and 2nd days after lentiviralmediated gene transfection. On the 4th and 8th days, SCAP-Foxc2 showed the highest proliferation level in the four groups $(P<0.05)$. On the 8th day, SCAP-Foxc2-BMP2 displayed the weakest proliferation status $(P<0.05)$.

3.4. Foxc2 Potentiated BMP2-Induced Osteo-/Odontogenic Differentiation of SCAP. The relative mRNA expression of ALP, OCN, and DSPP in SCAP-Foxc2-BMP2 showed a rising trend and achieved the peak expression on day 16 after transfection; however, the expression of DMP1 achieved the peak expression on day 8. Moreover, the mRNA expressions of ALP, OCN, DSPP, and DMP1 in SCAP-Foxc2-BMP2 were significantly higher than those in other groups at most of the time points $(P<0.05) \quad$ (Figure $4(\mathrm{a}))$. Western blot also showed that the protein expression of DSP in SCAPFoxc2-BMP2 was significantly higher than that in the other three groups at the four time points $(P<0.01)$ (Figures 4(b) and 4(c)).

3.5. ALP Staining of the Gene-Modified SCAP. These mineralized nodules with positive ALP expression were gold in color (Figure 5(a)). The number of mineralized nodules in SCAP-Foxc2-BMP2 was significantly more than that in the other three groups $(P<0.01)$. The number of mineralized nodules formed by SCAP-BMP2 was significantly more than that formed by SCAP-foxc2 and SCAP-GFP $(P<0.05)$ (Figure $5(b))$. The number of mineralized nodules of SCAP-Foxc2 was slightly more than that of SCAP-GFP, but there was no statistical significance between the two groups.

3.6. Alizarin Red Staining of the Gene-Modified SCAP. The mineralized nodules were stained as red (Figure 6(a)). The positive staining area and mineralized nodules' number of SCAP-Foxc2-BMP2 were significantly better than those of the other three group cells (Figure 6(b)). The mineralized nodules' number in SCAP-BMP2 was obviously more than that in SCAP-Foxc2, and the mineralized nodules' number in SCAP-Foxc2 was slightly more than that in SCAP-GFP.

\section{Discussion}

Dental apical papilla directly contributes toward the formation of the tooth roots [23]. As a type of multipotential mesenchymal stem cells derived from the apical papilla, SCAP can differentiate into osteoblasts, odontoblasts, adipocytes, and chondrocytes [24]. In the current research, those mesenchymal cells isolated from the apical papilla formed significant alizarin red-positive mineralized nodes and oil red O-positive lipid droplets after 

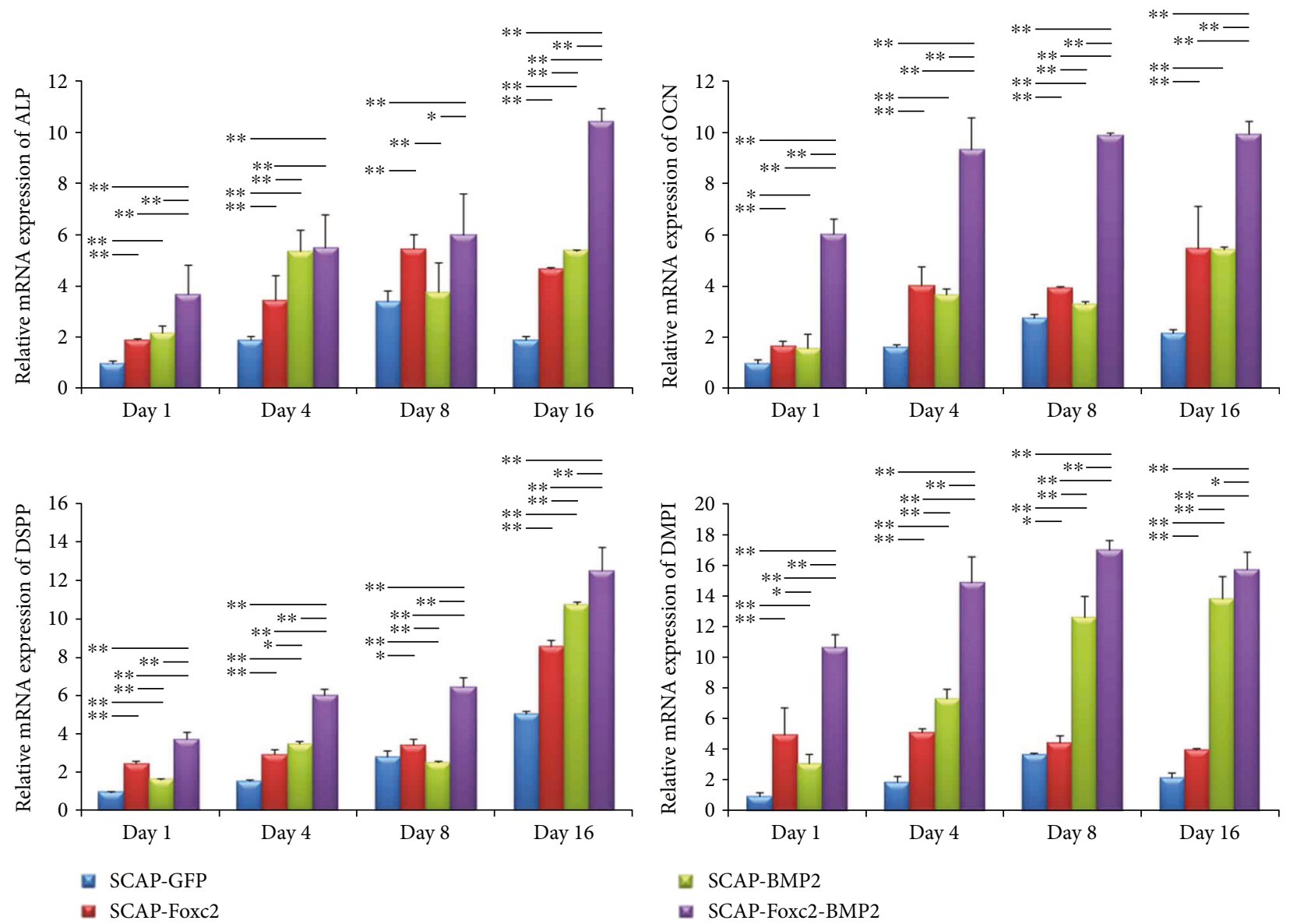

(a)

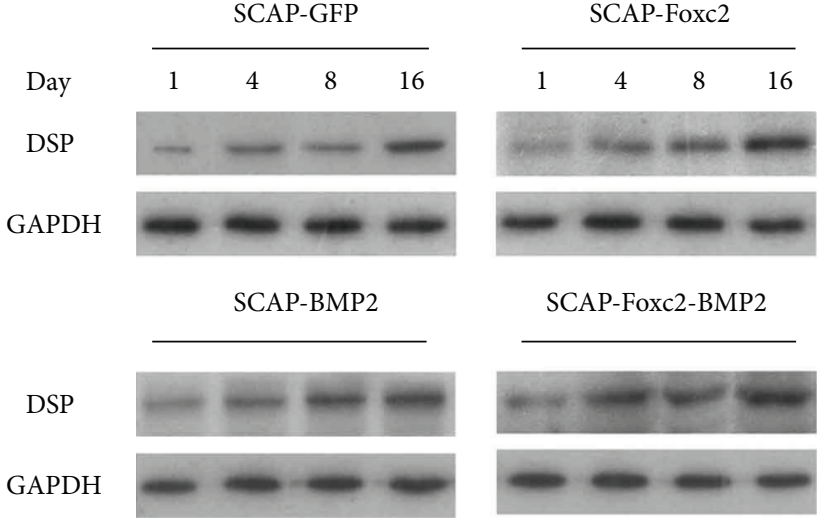

(b)

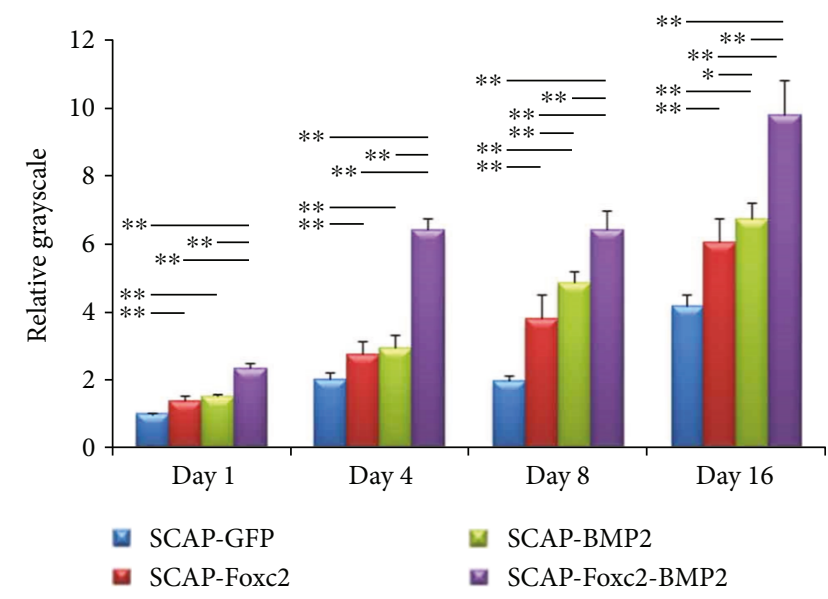

(c)

FIGURE 4: Relative expression of osteo-/odontogenic differentiation of mRNAs and proteins in the four group cells. (a) In SCAP-Foxc2-BMP2, the mRNA expression of ALP, OCN, DSPP, and DMP1 was significantly higher than that of the other groups at majority of the time points $(P<0.05)$. Specifically, the expression exhibited a rising trend over time. $(b, c)$ The DSP expression in SCAP-Foxc2-BMP2, which was detected by relative grayscale of western blot results, was significantly higher than that in the other groups at most of the time points $(P<0.05)$. The expression of DSP in SCAP-Foxc2-BMP2 also displayed a rising trend over time. ${ }^{*} P<0.05,{ }^{* *} P<0.01$.

induction in vitro. Moreover, those isolated cells presented typical phenotype of SCAP [24], which was verified by STRO-1(+), CD146(+), CD24(+), and CD45(-). Based on the above results, the mesenchymal cells isolated from the apical papilla in the current study were confirmed to be SCAP.

Foxc2 gene, which is necessary for mesenchymalepithelial interactions during craniofacial development 
SCAP-GFP

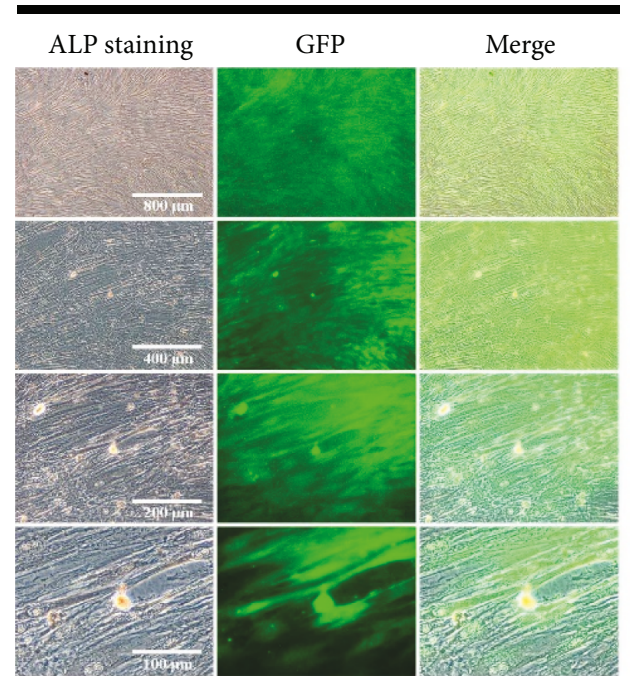

SCAP-BMP2

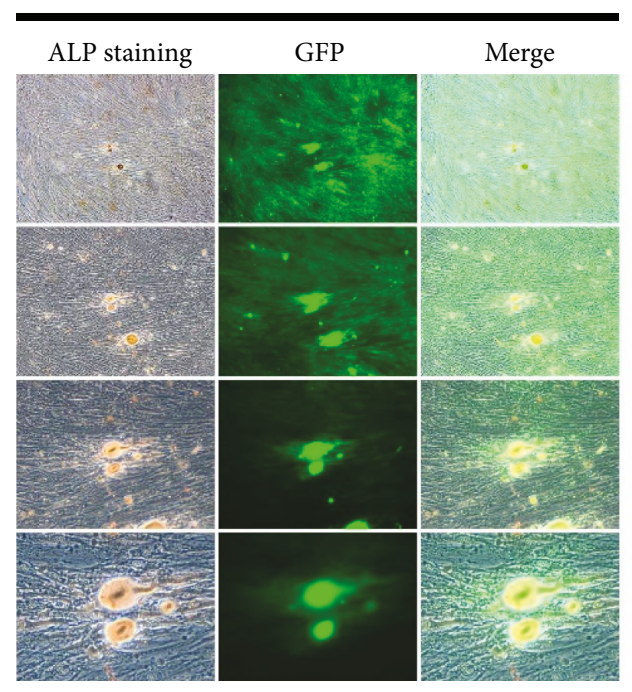

SCAP-Foxc2

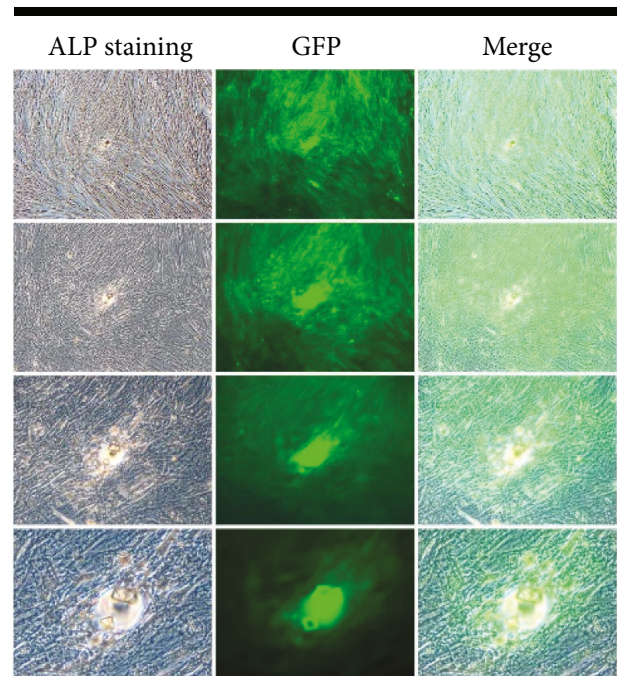

SCAP-Foxc2-BMP2

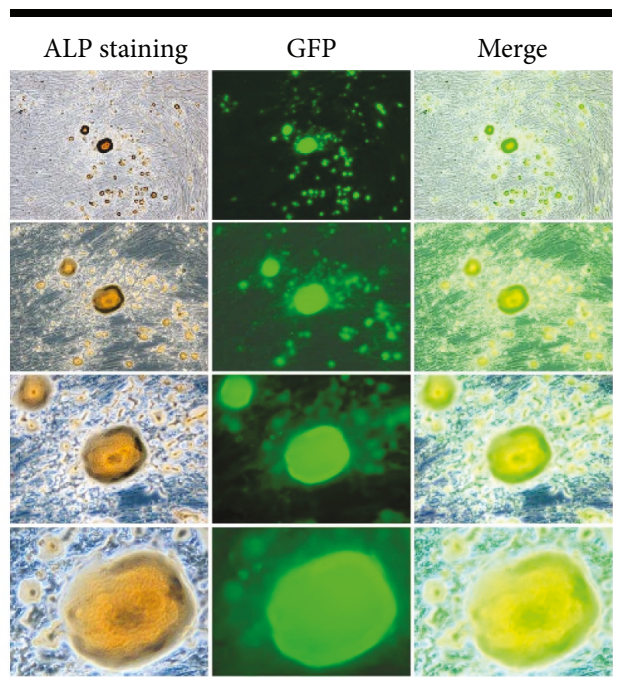

(a)

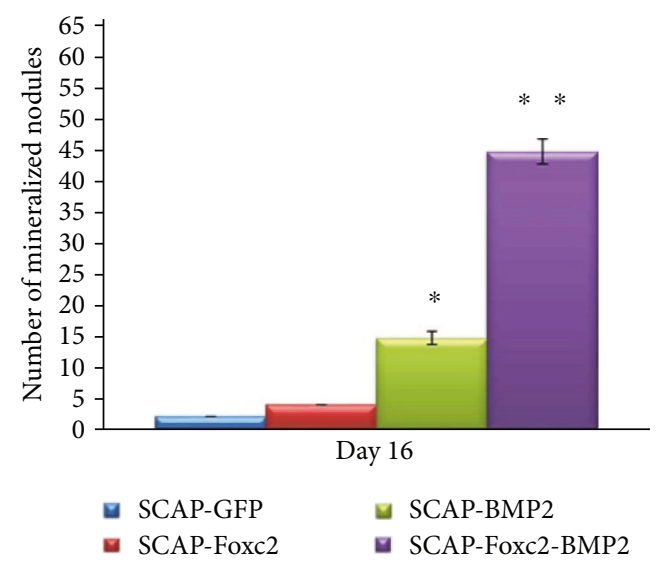

(b)

FIgURE 5: ALP staining status. (a) Those ALP-positive mineralized granules were stained as gold in color. (b) Those mineralized nodules formed by SCAP-Foxc2-BMP2 were significantly more than those formed by the other groups $(P<0.01)$. The mineralized nodules formed by SCAP-BMP2 was significantly more than those formed by SCAP-foxc2 and SCAP-GFP $(P<0.05)$, and mineralized nodules formed by SCAP-Foxc2 was slightly more than those formed by SCAP-GFP. ${ }^{*} P<0.05,{ }^{* *} P<0.01$. 


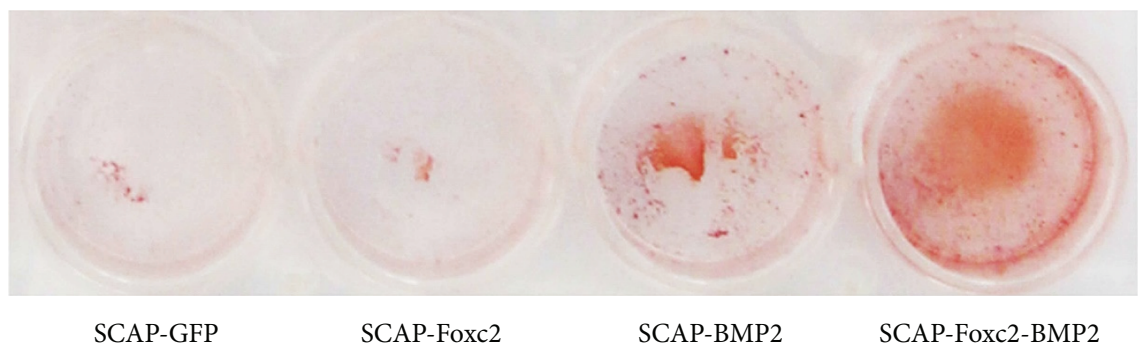

(a)

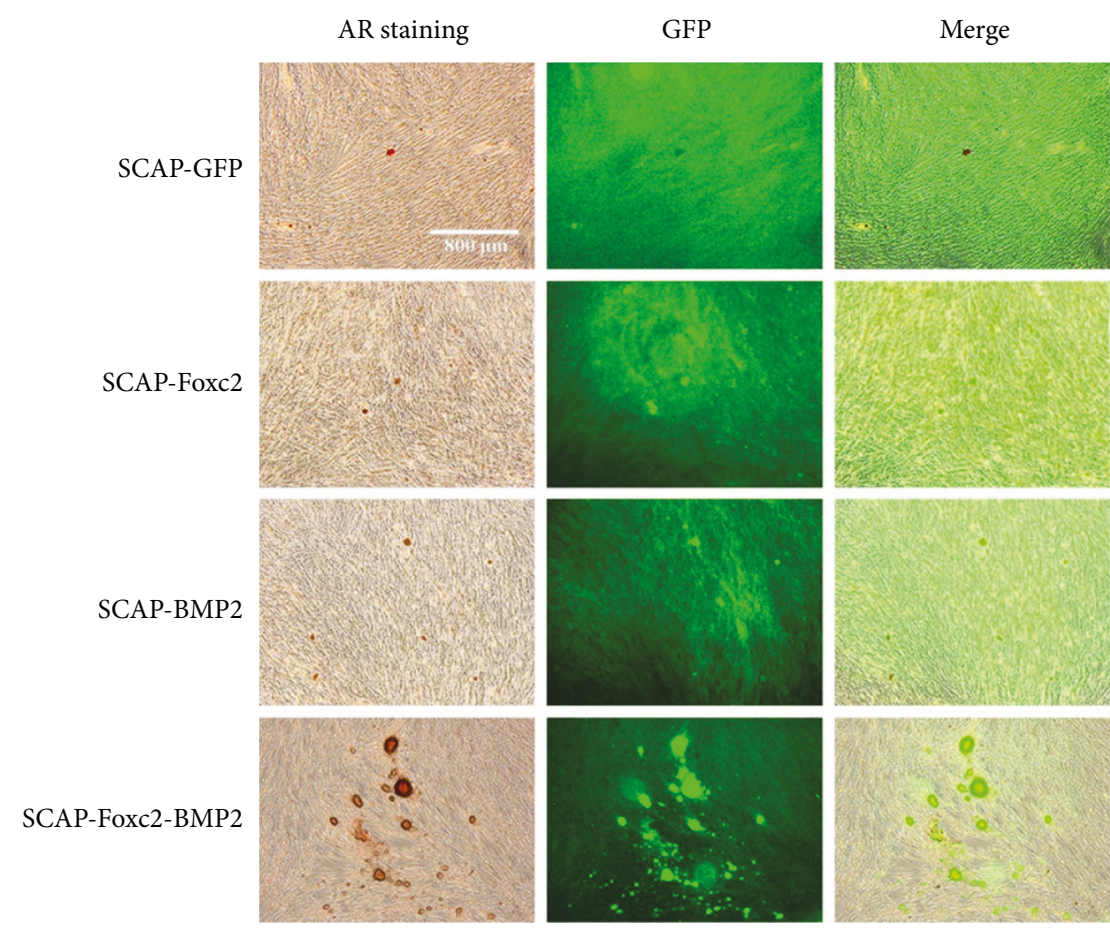

(b)

Figure 6: Alizarin red staining status. (a) The mineralized nodules were stained as red. (b) The positive staining area and mineralized nodules' number of SCAP-Foxc2-BMP2 were significantly better than those of the other three group cells.

[25-27], has been detected in mesodermal and neural crest derivatives [28]. As a mesenchymal tissue derived from neural crest, the dental apical papilla also expressed Foxc2 [19]. Studies pointed out that BMP proteins and osteogenic induction culture medium could significantly increase the expression of Foxc2 in bone mesenchymal stem cells, dental follicle stem cells, skeletal precursor cells, and amniotic fluid-derived mesenchymal stem cells $[15,20,29,30]$. The study indicates that Foxc2 is a signal of canonical Wnt pathway in mouse P19 embryonal carcinoma cells [31]. In the current study, Foxc2 gene expression in the BMP2-modified SCAP was slightly increased, but upregulated Foxc2 gene expression did not significantly boost the expression of BMP2 in SCAP. This result suggests that Foxc2 expression is regulated by BMP2 in SCAP, and Foxc2 might be a downstream signal of BMP2. Several scientists have investigated the molecular mechanism of Foxc2 in promoting osteogenic differentiation. Gozo et al. [13] found that the overexpression of Foxc2 can upregulate
Wnt4 and BMP4 in osteoblasts. Park et al. [11] suggested that the overexpression of Foxc2 stimulated osteogenic differentiation of osteoblasts by increasing the expression level of integrin $\beta 1$. Kim et al. [18] reported that the overexpression of Foxc2 increased bone formation through $\beta$-catenin pathway. In the current research, the Foxc2 gene-modified SCAP upregulated the expression of osteo-/odontogenic markers, including ALP, OCN, DSPP, and DMP1. Similar phenomenon was also observed in other Foxc2-modified cells, such as bone marrow mesenchymal stem cells $[14,30]$, myoblasts [13], and osteoblasts [18].

Furthermore, our research presented that lentivirusmediated Foxc2 gene transfection improved the ability of SCAP to form mineralized nodules in vitro, indicating that Foxc2 gene participated in regulating of mineralization process. Additionally, the mineralization capability of SCAP-Foxc2-BMP2 was significantly stronger than that of the other three groups. Mineralized nodules formed 
by SCAP-Foxc2-BMP2 cells were about 3 times more than those of SCAP-BMP2, about eleven times more than those of SCAP-Foxc2, and about twenty-two times more than those of SCAP-GFP. These results suggest that Foxc2 and BMP2 synergistically promote osteo-/odontogenic differentiation and mineralization of SCAP.

A few studies discovered that Foxc2 could stimulate proliferation of some cells. In myoblasts, decreased endogenous Foxc2 reduced cell proliferation activity, while increased expression of Foxc2 promoted cell proliferation [13]. In osteoblasts, upregulated Foxc2 facilitates cell proliferation in vitro [32], and Foxc2 might encourage the survival and proliferation of osteoblasts by upregulating integrin $\beta 1$ [11]. Consistent with the previous reports $[13,32]$, our data showed that the proliferation of Foxc2 gene-transfected SCAP was slightly stronger than that of the other three groups. However, the proliferative activity of Foxc2 and BMP2 double gene-transfected SCAP was reduced on day 8, which implies that Foxc2 and BMP2 synergistically control the proliferation of SCAP. Some studies pointed out that Foxc2 promoted proliferation by activating mTORC1 in preadipocytes [12] and mesenchymal stem cells [33]. However, in osteoblasts, mTORC1 was activated by BMP2 to promote osteogenic differentiation [34]. So, it is speculated that the function of mTORC1 may be changed from promoting cell proliferation to enhancing cell osteo-/odontogenic differentiation when Foxc2 and BMP2 were both activated in SCAP.

In summary, our research indicated that Foxc2 and BMP2 genes could act synergistically to improve osteo-/ odontogenic differentiation and mineralization of SCAP in vitro. These results have a positive impact on the clinical practice of tooth regeneration. The future study could focus on the interaction between these two genes and the mechanism of their cooperative function on SCAP. Moreover, in vivo studies could further support this discovery.

\section{Data Availability}

The data used to support the findings of this study are available from the corresponding author upon request.

\section{Conflicts of Interest}

The authors declare that they have no competing interests.

\section{Acknowledgments}

This study was supported by the National Natural Science Foundation of China (81500825, 81470731, 81602384, and 11772361), Natural Science Foundation of Guangdong Province (2016A030313275), Medical Scientific Research Foundation of Guangdong Province (201512220616175), Special Fund for Public Welfare Research and Capacity Building of Guangdong Province (2014A020212211), and Science and Technology Program of Guangzhou (201607010205).

\section{References}

[1] T. Gong, B. C. Heng, E. C. M. Lo, and C. Zhang, "Current advance and future prospects of tissue engineering approach to dentin/pulp regenerative therapy," Stem Cells International, vol. 2016, Article ID 9204574, 13 pages, 2016.

[2] J. Liu, F. Yu, Y. Sun et al., "Concise reviews: characteristics and potential applications of human dental tissue-derived mesenchymal stem cells," Stem Cells, vol. 33, no. 3, pp. 627-638, 2015.

[3] A. Bakopoulou and I. About, "Stem cells of dental origin: current research trends and key milestones towards clinical application," Stem Cells International, vol. 2016, Article ID 4209891, 20 pages, 2016.

[4] W. Zhang, X. Zhang, J. Ling, X. Wei, and Y. Jian, "Osteo -/odontogenic differentiation of BMP2 and VEGF gene-cotransfected human stem cells from apical papilla," Molecular Medicine Reports, vol. 13, no. 5, pp. 3747-3754, 2016.

[5] H. Zhang, J. Wang, F. Deng et al., "Canonical Wnt signaling acts synergistically on BMP9-induced osteo/odontoblastic differentiation of stem cells of dental apical papilla (SCAPs)," Biomaterials, vol. 39, pp. 145-154, 2015.

[6] W. Zhang, X. Zhang, J. Ling et al., "Proliferation and odontogenic differentiation of BMP2 genetransfected stem cells from human tooth apical papilla: an in vitro study," International Journal of Molecular Medicine, vol. 34, no. 4, pp. 1004-1012, 2014.

[7] A. Balic and I. Thesleff, "Tissue interactions regulating tooth development and renewal," Current Topics in Developmental Biology, vol. 115, pp. 157-186, 2015.

[8] M. Jussila and I. Thesleff, "Signaling networks regulating tooth organogenesis and regeneration, and the specification of dental mesenchymal and epithelial cell lineages," Cold Spring Harbor Perspectives in Biology, vol. 4, no. 4, article a008425, 2012.

[9] W. Wang, M. Dang, Z. Zhang et al., "Dentin regeneration by stem cells of apical papilla on injectable nanofibrous microspheres and stimulated by controlled BMP-2 release," Acta Biomaterialia, vol. 36, pp. 63-72, 2016.

[10] L. A. Wu, J. Feng, L. Wang et al., "Immortalized mouse floxed Bmp2 dental papilla mesenchymal cell lines preserve odontoblastic phenotype and respond to BMP2," Journal of Cellular Physiology, vol. 225, no. 1, pp. 132-139, 2010.

[11] S. J. Park, J. Gadi, K. W. Cho et al., “The forkhead transcription factor Foxc2 promotes osteoblastogenesis via up-regulation of integrin $\beta 1$ expression," Bone, vol. 49, no. 3, pp. 428-438, 2011.

[12] L. Gan, Z. Liu, W. Jin, Z. Zhou, and C. Sun, "Foxc2 enhances proliferation and inhibits apoptosis through activating Akt/ mTORC1 signaling pathway in mouse preadipocytes," Journal of Lipid Research, vol. 56, no. 8, pp. 1471-1480, 2015.

[13] M. C. Gozo, P. J. Aspuria, D. J. Cheon et al., "Foxc2 induces Wnt4 and Bmp4 expression during muscle regeneration and osteogenesis," Cell Death \& Differentiation, vol. 20, no. 8, pp. 1031-1042, 2013.

[14] W. You, H. Gao, L. Fan, D. Duan, C. Wang, and K. Wang, "Foxc2 regulates osteogenesis and angiogenesis of bone marrow mesenchymal stem cells," BMC Musculoskeletal Disorders, vol. 14, no. 1, p. 199, 2013.

[15] A. Nifuji, N. Miura, N. Kato, O. Kellermann, and M. Noda, "Bone morphogenetic protein regulation of forkhead/winged helix transcription factor Foxc2 (Mfh1) in a murine 
mesodermal cell line C1 and in skeletal precursor cells," Journal of Bone and Mineral Research, vol. 16, no. 10, pp. 1765-1771, 2001.

[16] T. Kume, "Foxc2 transcription factor: a newly described regulator of angiogenesis," Trends in Cardiovascular Medicine, vol. 18, no. 6, pp. 224-228, 2008.

[17] W. You, K. Wang, D. Duan, C. Wang, L. Fan, and R. Liu, "Construction of lentiviral vector containing Homo sapiens forkhead box C2 gene and its expression in bone marrow mesenchymal stem cells of rabbits," Zhongguo Xiu Fu Chong Jian Wai Ke Za Zhi, vol. 27, no. 5, pp. 535-540, 2013.

[18] S. H. Kim, K. W. Cho, H. S. Choi et al., "The forkhead transcription factor Foxc2 stimulates osteoblast differentiation," Biochemical and Biophysical Research Communications, vol. 386, no. 3, pp. 532-536, 2009.

[19] M. Gosau, W. Götz, O. Felthaus, T. Ettl, A. Jäger, and C. Morsczeck, "Comparison of the differentiation potential of neural crest derived progenitor cells from apical papilla (dNC-PCs) and stem cells from exfoliated deciduous teeth (SHED) into mineralising cells," Archives of Oral Biology, vol. 58, no. 6, pp. 699-706, 2013.

[20] T. Vollkommer, M. Gosau, O. Felthaus, T. E. Reichert, C. Morsczeck, and W. Götz, "Genome-wide gene expression profiles of dental follicle stem cells," Acta Odontologica Scandinavica, vol. 73, no. 2, pp. 93-100, 2015.

[21] K. Uchibe, H. Shimizu, S. Yokoyama, T. Kuboki, and H. Asahara, "Identification of novel transcription-regulating genes expressed during murine molar development," Developmental Dynamics, vol. 241, no. 7, pp. 1217-1226, 2012.

[22] W. Zhang, W. Liu, J. Ling et al., "Odontogenic differentiation of vascular endothelial growth factor-transfected human dental pulp stem cells in vitro," Molecular Medicine Reports, vol. 10, no. 4, pp. 1899-1906, 2014.

[23] G. T. J. Huang, W. Sonoyama, Y. Liu, H. Liu, S. Wang, and S. Shi, "The hidden treasure in apical papilla: the potential role in pulp/dentin regeneration and bioroot engineering," Journal of Endodontia, vol. 34, no. 6, pp. 645-651, 2008.

[24] G. T. J. Huang, S. Gronthos, and S. Shi, "Mesenchymal stem cells derived from dental tissues vs. those from other sources: their biology and role in regenerative medicine," Journal of Dental Research, vol. 88, no. 9, pp. 792-806, 2009.

[25] S. Seo, L. Chen, W. Liu et al., "Foxc1 and Foxc2 in the neural crest are required for ocular anterior segment development," Investigative Ophthalmology \& Visual Science, vol. 58, no. 3, pp. 1368-1377, 2017.

[26] M. Motojima, S. Tanimoto, M. Ohtsuka, T. Matsusaka, T. Kume, and K. Abe, "Characterization of kidney and skeleton phenotypes of mice double heterozygous for Foxc1 and Foxc2," Cells Tissues Organs, vol. 201, no. 5, pp. 380-389, 2016.

[27] M. B. Amin, N. Miura, M. K. M. Uddin et al., "Foxc2 ${ }^{C r E E R T 2}$ knock-in mice mark stage-specific Foxc2-expressing cells during mouse organogenesis," Congenital Anomalies, vol. 57, no. 1, pp. 24-31, 2017.

[28] T. Kume, "The cooperative roles of Foxc1 and Foxc2 in cardiovascular development," in Forkhead Transcription Factors, vol. 665 of Advances in Experimental Medicine and Biology, pp. 63-77, 2009.

[29] J. Si, J. Dai, J. Zhang et al., "Comparative investigation of human amniotic epithelial cells and mesenchymal stem cells for application in bone tissue engineering," Stem Cells International, vol. 2015, Article ID 565732, 14 pages, 2015.
[30] W. You, L. Fan, D. Duan et al., "Foxc2 over-expression in bone marrow mesenchymal stem cells stimulates osteogenic differentiation and inhibits adipogenic differentiation," Molecular and Cellular Biochemistry, vol. 386, no. 1-2, pp. 125-134, 2014.

[31] J. Savage, A. Voronova, V. Mehta, F. Sendi-Mukasa, and I. S. Skerjanc, "Canonical Wnt signaling regulates Foxc1/2 expression in P19 cells," Differentiation, vol. 79, no. 1, pp. 31-40, 2010.

[32] Z. Wang, C. Fu, Y. Chen et al., "FoxC2 enhances BMP7mediated anabolism in nucleus pulposus cells of the intervertebral disc," PLoS One, vol. 11, no. 1, article e0147764, 2016.

[33] H. J. Lee, J. M. Ryu, Y. H. Jung, S. Y. Oh, S. J. Lee, and H. J. Han, "Novel pathway for hypoxia-induced proliferation and migration in human mesenchymal stem cells: involvement of HIF-1 $\alpha$, FASN, and mTORC1," Stem Cells, vol. 33, no. 7, pp. 2182-2195, 2015.

[34] C. M. Karner, S. Y. Lee, and F. Long, "Bmp induces osteoblast differentiation through both Smad4 and mTORC1 signaling," Molecular and Cellular Biology, vol. 37, no. 4, pp. e00253e00216, 2017. 


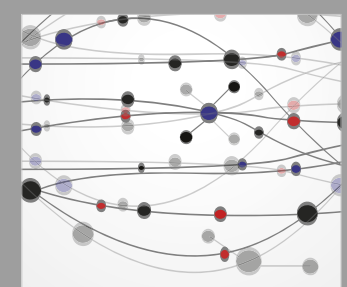

The Scientific World Journal
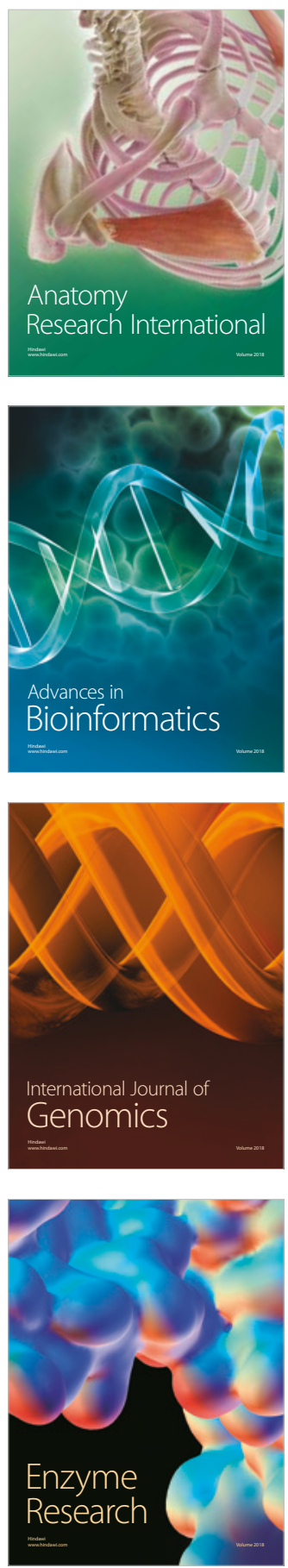
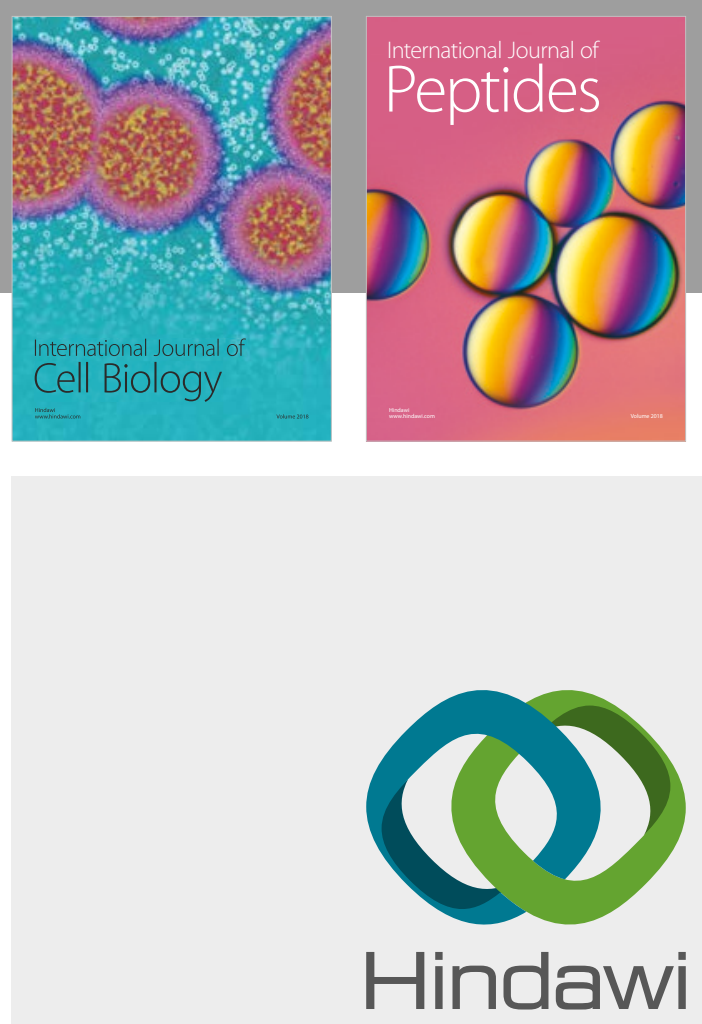

Submit your manuscripts at

www.hindawi.com
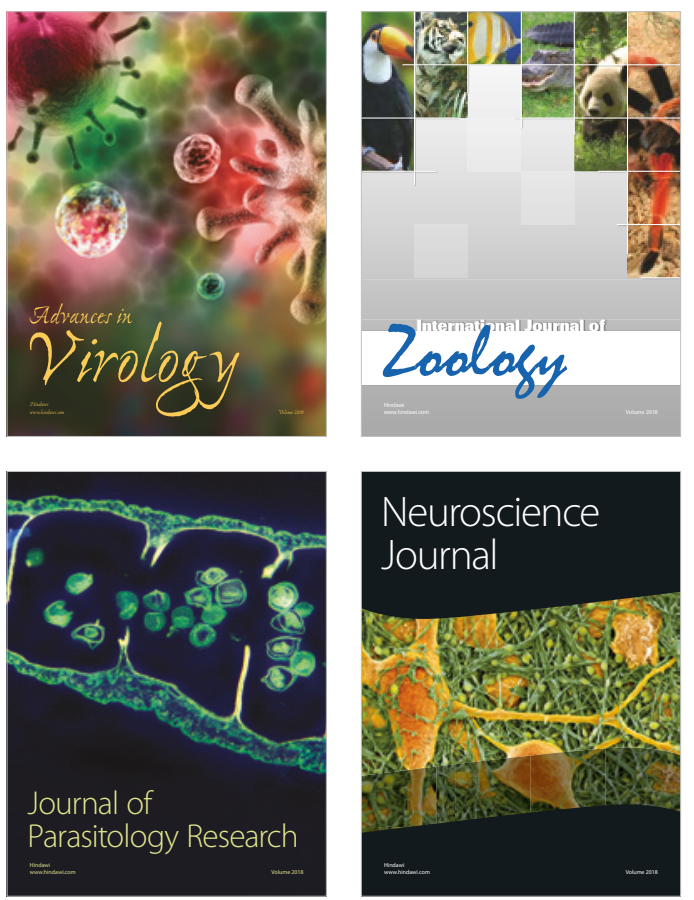
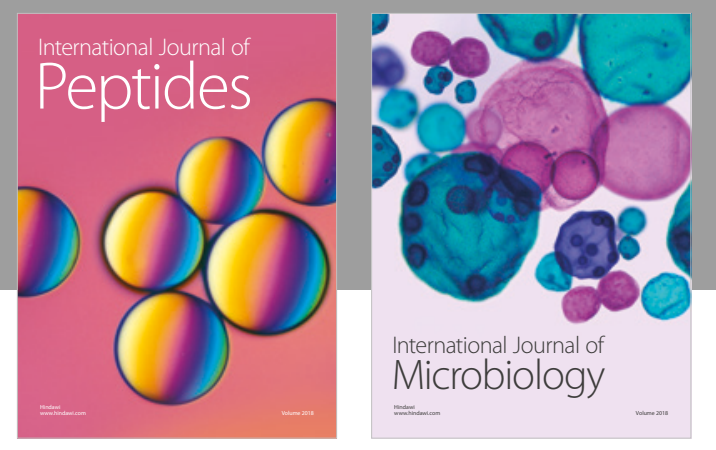

nternational Journal of Microbiology
Journal of
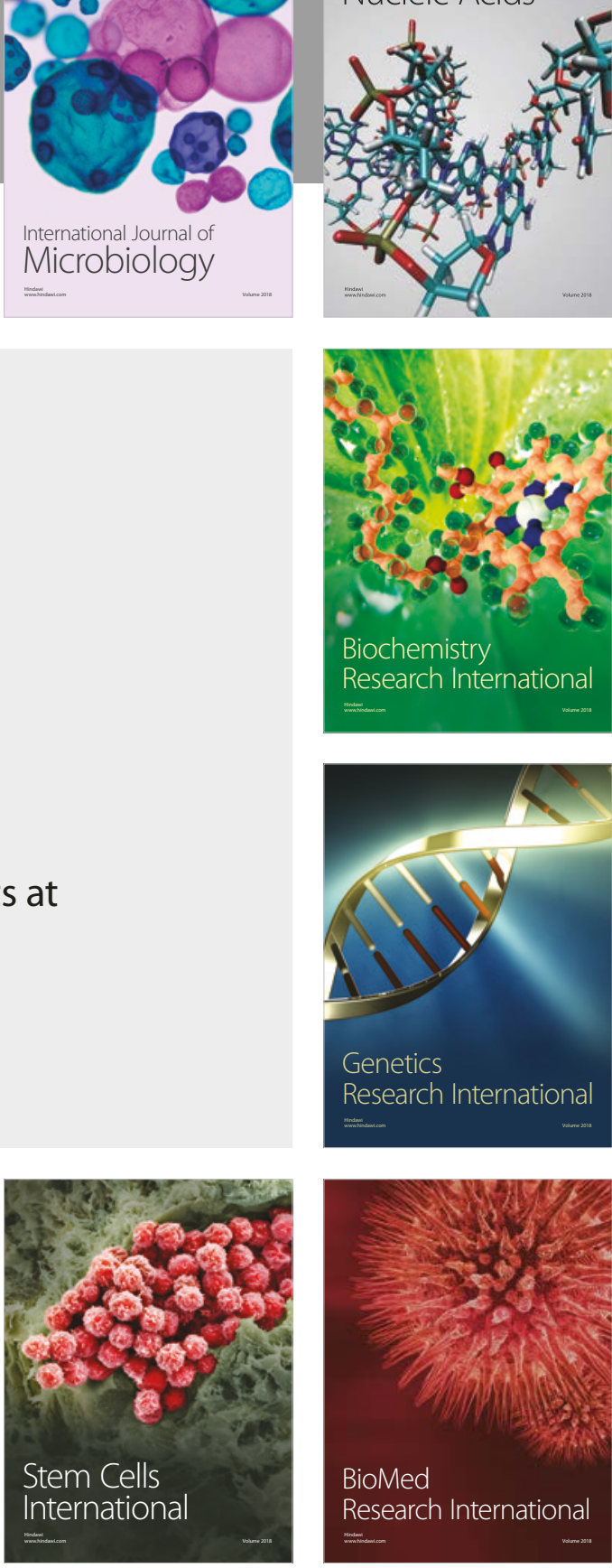
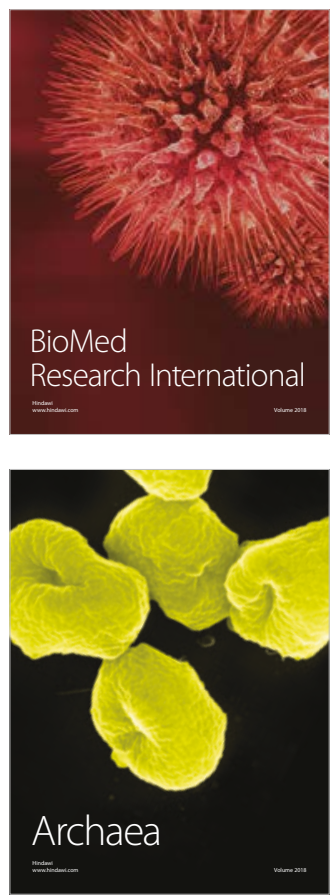